\begin{tabular}{|c|c|}
\hline Title & Isotope scattering of large wave-vector phonons in GaA s and InSb : Deformation-dipole and overlap-shell models \\
\hline Author(s) & Tamura, Shin-ichiro \\
\hline Citation & $\begin{array}{l}\text { Physical Review B, 30(2), 849-854 } \\
\text { https://doi.org/10.1103/PhysRevB.30.849 }\end{array}$ \\
\hline Issue Date & $1984-07-15$ \\
\hline Doc URL & http:/hdl .handle.net/2115/5954 \\
\hline Rights & Copyright $\odot 1984$ A merican Physical Society \\
\hline Type & article \\
\hline File Information & PRB30-2.pdf \\
\hline
\end{tabular}

Instructions for use 


\title{
Isotope scattering of large-wave-vector phonons in GaAs and InSb: Deformation-dipole and overlap-shell models
}

\author{
Shin-ichiro Tamura \\ Department of Engineering Science, Hokkaido University, Sapporo 060, Japan
}

(Received 23 March 1984)

\begin{abstract}
Scatterings of near-zone-boundary phonons by isotopic disorder in diatomic GaAs and InSb crystals are studied theoretically. In multiatomic lattices the scattering rate depends importantly on the vibrational amplitudes of constituent atoms. We find that the wave-vector dependences of these amplitudes, which are predicted by some lattice models, show quite dissimilar behaviors as compared to others. The typical examples investigated in this paper are the deformation-dipole model and the overlap-shell model. With the use of these models the frequency dependence of the relaxation time of phonons is calculated. Both models, however, predict the relaxation time of about $0.1 \mu \mathrm{sec}$ for 1$\mathrm{THz}$ TA phonons in GaAs, which is at least 1 order of magnitude shorter than the lifetime reported by Ulbrich et al.
\end{abstract}

\section{INTRODUCTION}

There has been a great deal of recent interest in the propagation of nonequilibrium, large-wave-vector phonons at low temperatures. ${ }^{1,2}$ For the moment, an important problem from the theoretical viewpoint may be how to understand the anomalously long lifetimes of acoustic phonons observed by Ulbrich, Narayanamurti, and Chin. ${ }^{3}$ They have reported that the near-zone-edge TA phonons generated by $e$ - $h$ recombination in high-purity GaAs possess lifetimes greater than $1 \mu \mathrm{sec}$ and propagate ballistically over millimeter distances at dispersive velocities. Theoretical predictions on the anharmonic decay of highenergy phonons satisfying $\hbar \omega \gg k_{B} T$ ( $\omega$ being the angular frequency of phonons and $T$ the ambient temperature) indeed show very long energy-relaxation times for TA phonons. ${ }^{4-6}$ In contrast, high-frequency phonons in isotopically impure crystals are scattered quite frequently by fluctuations in the mass distribution of atoms, leading, in general, to very short momentum-relaxation times. ${ }^{7}$

Recently, Lax et al. ${ }^{8}$ have shown theoretically that the isotope-scattering rate of phonons in diatomic crystals such as GaAs which have one element (As) isotopically pure depends strongly on the amplitude of vibration of the impure element $(\mathrm{Ga})$. Based on the bond-change model ${ }^{9}$ (BCM) they demonstrated the wavelength dependence of the amplitude of $\mathrm{Ga}$ motion associated with acoustic modes and then suggested the existence of some frequency window over which the isotope scattering may be weaker than that expected from the theory valid for the monoatomic case.

Here we remark that, as pointed out by Lax et al. ${ }^{8}$ the isotope scattering in multiatomic lattices depends explicitly on the eigenvectors of atomic displacements (see next section), and hence it will, in principle, provide a stringent test for lattice models. This is because for a complete description of lattice dynamics, the model in question should not only satisfy the experimental eigenfrequencies, but also reproduce the correct eigenvectors of atomic motions in the entire Brillouin zone. ${ }^{10}$
The purpose of this paper is to closely investigate the isotope scattering of large-wave-vector phonons in GaAs and InSb in connection with the experiment by Ulbrich et $a l^{3}$ as well as with the validity of some lattice models. We consider InSb since it is much more dispersive than GaAs and is characterized by the lowest zone-boundary frequency, of about $1.0 \mathrm{THz},{ }^{11}$ whereas this frequency amounts to about $1.9 \mathrm{THz}$ (Ref. 12) for GaAs. Throughout this work we employ two distinct models, i.e., the deformation-dipole model ${ }^{13,14}$ (DDM) and the valence-overlap-shell model ${ }^{15-17}$ (VOSM), for which partial trend analyses of the lattice dynamics of many compounds with zinc-blende structure exist. As we shall see later these two models show quite dissimilar behaviors to each other regarding the wavelength dependence of atomic displacements, which should yield large differences in the isotope-scattering rates of near-zone-boundary phonons. The resulting relaxation times predicted by the DDM and VOSM indeed differ remarkably near the zone boundaries, which, in principle, could be discriminated from one another by experiments.

In the next section we give the expression for the scattering rate of phonons by isotopes. Comparing it with the formula obtained in the low-frequency limit we stress the finite-frequency effects (dispersive effects) on the scattering. In Sec. III the properties of phonons in GaAs and InSb predicted by the DDM and VOSM are described. The emphasis is placed on elucidating the characteristic behaviors of polarization vectors predicted by these models. The numerical results on the relaxation time of phonons are given in Sec. IV and their implications to the experiment by Ulbrich et $a l^{3}$ are also discussed.

\section{SCATTERING RATE}

In a crystal in which isotopic atoms are distributed randomly on the lattice sites, the scattering rate, or the reciprocal of the relaxation time $\tau$ of a phonon $(\vec{q}, j)(\vec{q}$ is 
the wave vector and $j$ specifies the phonon mode) scattered by isotopes, is given by ${ }^{18}$

$$
\begin{aligned}
\tau^{-1}(\overrightarrow{\mathrm{q}}, j ; \omega)= & \frac{\pi}{2 N} \omega^{2}(\overrightarrow{\mathrm{q}}, j) \\
& \times \sum_{\overrightarrow{\mathrm{q}}^{\prime}, j^{\prime}} \delta\left(\omega-\omega\left(\overrightarrow{\mathrm{q}}^{\prime}, j^{\prime}\right)\right) \\
& \quad \times \sum_{\sigma} g(\sigma)\left|\overrightarrow{\mathrm{e}}^{*}\left(\sigma \mid \overrightarrow{\mathrm{q}}^{\prime}, j^{\prime}\right) \cdot \overrightarrow{\mathrm{e}}(\sigma \mid \overrightarrow{\mathrm{q}}, j)\right|^{2},
\end{aligned}
$$

where $N$ is the number of unit cells in the crystal and $\overrightarrow{\mathrm{e}}$ is the unit amplitude (polarization vector) of the lattice, which is determined by solving the eigenvalue equations ${ }^{19}$

$$
\begin{aligned}
& \sum_{\beta, \sigma^{\prime}}\left[\omega^{2} \delta_{\alpha \beta} \delta_{\sigma \sigma^{\prime}}-G_{\alpha \beta}\left(\sigma, \sigma^{\prime} \mid \overrightarrow{\mathrm{q}}\right)\right] e_{\beta}\left(\sigma^{\prime}\right)=0 \\
& \quad \text { for } \alpha=1,2,3, \quad \sigma=1,2, \ldots, r .
\end{aligned}
$$

Here, $G_{\alpha \beta}$ is the so-called dynamical matrix of the lattice and $r$ denotes the number of atoms in a unit cell. In Eq. (1), $g(\sigma)$ is the constant which represents the strength of isotope effects; it can be expressed in terms of mass $M_{i}(\sigma)$ and its fraction $f_{i}(\sigma)$ in the $i$ th isotope of $\sigma$ atom as

$$
g(\sigma)=\sum_{i} f_{i}(\sigma)\left[1-\frac{M_{i}(\sigma)}{\bar{M}(\sigma)}\right]^{2}
$$

where $\bar{M}(\sigma)$ is the average mass of $\sigma$ atom defined by

$$
\bar{M}(\sigma)=\sum_{i} f_{i}(\sigma) M_{i}(\sigma) .
$$

In a crystal of cubic symmetry we have

$$
\begin{aligned}
\sum_{\overrightarrow{\mathrm{q}}, j} F(\overrightarrow{\mathrm{q}}, j) e_{\alpha}^{*}(\sigma \mid \overrightarrow{\mathrm{q}}, j) e_{\beta}(\sigma \mid \overrightarrow{\mathrm{q}}, j) & \\
& =\frac{1}{3} \delta_{\alpha \beta} \sum_{\overrightarrow{\mathrm{q}}, j} F(\overrightarrow{\mathrm{q}}, j)|\overrightarrow{\mathrm{e}}(\sigma \mid \overrightarrow{\mathrm{q}}, j)|^{2},
\end{aligned}
$$

where $F$ is an arbitrary function which depends on $\vec{q}$ and $j$ through $\omega(\overrightarrow{\mathrm{q}}, j)$. Accordingly, Eq. (1) is simplified to

$$
\begin{aligned}
\tau^{-1}(\overrightarrow{\mathrm{q}}, j ; \omega)= & \frac{\pi}{6 N} \omega^{2}(\overrightarrow{\mathrm{q}}, j) \\
& \times \sum_{\sigma} g(\sigma)|\overrightarrow{\mathrm{e}}(\sigma \mid \overrightarrow{\mathrm{q}}, j)|^{2} \\
& \times \sum_{\overrightarrow{\mathrm{q}}^{\prime}, j^{\prime}} \delta\left(\omega-\omega\left(\overrightarrow{\mathrm{q}}^{\prime}, j^{\prime}\right)\right)\left|\overrightarrow{\mathrm{e}}\left(\sigma \mid \overrightarrow{\mathrm{q}}^{\prime}, j^{\prime}\right)\right|^{2} .
\end{aligned}
$$

Thus the scattering rate is described by the squared amplitudes of the atomic vibration associated with the initial phonon multiplied by the one-phonon density of states which is also weighted by the square of polarization vectors of the final phonon.

In the following we apply formula (6) to the scatterings in GaAs and InSb. In these cases the sum over $\sigma$ runs to $\sigma=1$ and 2, and the values of $g(\sigma)$ take $g(1)$ $=g_{\mathrm{Ga}}=1.97 \times 10^{-4}$ and $g(2)=g_{\mathrm{As}}=0$ for GaAs, and $g(1)=g_{\text {In }}=1.23 \times 10^{-5}$ and $g(2)=g_{\mathrm{Sb}}=6.61 \times 10^{-5}$ for
InSb. Hence, the atomic vibration of the heavier element, As, does not contribute to the scattering of phonons in GaAs, but phonons may be scattered predominantly by the heavier element, $\mathrm{Sb}$, in InSb.

For the sake of comparison we shall explicitly write the scattering rate (6) for monoatomic lattices. In this case $g(\sigma)$ does not depend on $\sigma$, and then setting $g(\sigma)=g$ we obtain

$$
\tau_{\text {mono }}^{-1}(\overrightarrow{\mathrm{q}}, j ; \omega)=\frac{\pi}{12 N} g \omega^{2}(\overrightarrow{\mathrm{q}}, j) \sum_{\overrightarrow{\mathrm{q}}^{\prime}, j^{\prime}} \delta\left(\omega-\omega\left(\overrightarrow{\mathrm{q}}^{\prime}, j^{\prime}\right)\right) .
$$

Important features observed from Eqs. (6) and (7) may be summarized as follows. First, the isotope scattering of phonons in monoatomic crystals is governed by the ordinary one-phonon density of states and has no spatial anisotropy and polarization-vector dependence. Second, in multiatomic crystals it is the weighted one-phonon density of states which serves as available density of terminal states for scattered phonons and the scattering rate depends strongly on the polarization vector of initial phonons. Taking these results into account together with the fact that As is an isotopically pure element, Lax et al. ${ }^{8}$ have suggested the presence of some frequency window for the isotope scattering in GaAs.

In order to evaluate the dispersive effects on the isotope scattering, here we deduce the expression for the scattering rate in the low-frequency limit. In this limit the amplitudes of vibration for acoustic branches $[\omega(\overrightarrow{\mathrm{q}} \rightarrow \overrightarrow{0}, j) \rightarrow 0, j=1,2,3]$ are characterized by the relations $^{19}$

$$
|\overrightarrow{\mathrm{e}}(\sigma \mid \overrightarrow{\mathrm{q}} \rightarrow \overrightarrow{0}, j)|^{2} \propto \bar{M}(\sigma), \quad j=1,2,3 .
$$

Then Eq. (6) is reduced to

$$
\begin{array}{r}
\tau^{-1}(\overrightarrow{\mathrm{q}} \rightarrow \overrightarrow{0}, j ; \omega)=\frac{\pi}{6 N} \widetilde{g} \sum_{\overrightarrow{\mathrm{q}}^{\prime}, j^{\prime}} \delta\left(\omega-\omega\left(\overrightarrow{\mathrm{q}}^{\prime}, j^{\prime}\right)\right), \\
j=1,2,3,
\end{array}
$$

where

$$
\widetilde{g}=\sum_{\sigma} g(\sigma) \bar{M}^{2}(\sigma) / \sum_{\sigma} \bar{M}^{2}(\sigma),
$$

and the summation over $j^{\prime}$ of Eq. (9) should be taken over the acoustic branches (this is indicated by the "prime" on the summation sign). Thus the apparent polarizationvector dependence of the scattering rate of Eq. (6) disappears and spatial isotropy is recovered for the scattering of acoustic phonons in the long-wavelength limit.

Now, the one-phonon density of states $D(\omega)$ per unit cell defined by

$$
D(\omega)=\frac{1}{N} \sum_{\overrightarrow{\mathrm{q}}^{\prime}, j^{\prime}}^{\prime} \delta\left(\omega-\omega\left(\overrightarrow{\mathrm{q}}^{\prime}, j^{\prime}\right)\right)
$$

can be calculated in the low-frequency limit by the formula 


$$
\begin{aligned}
D(\omega \rightarrow 0)=\frac{V_{0}}{(2 \pi)^{3}} \omega^{2} \sum_{j^{\prime}}^{\prime} \int d \Omega\left(\overrightarrow{\mathrm{q}}^{\prime}, j^{\prime}\right) & \\
& \times\left.\frac{1}{v^{3}\left(\overrightarrow{\mathrm{q}}^{\prime}, j^{\prime}\right)}\right|_{\omega\left(\overrightarrow{\mathrm{q}}^{\prime}, j^{\prime}\right)=\omega},
\end{aligned}
$$

with the published values of elastic constants ${ }^{20,21}$ and mass density, where $V_{0}$ is the volume of a unit cell and $v$ is the phase velocity of the phonons. After numerical integrations of Eq. (12) we find, for $v=\omega / 2 \pi \rightarrow 0$,

$$
\tau_{\mathrm{GaAs}}^{-1}=7.38 \times 10^{-42} v^{4}, \quad \tau_{\mathrm{InSb}}^{-1}=1.62 \times 10^{-41} v^{4},
$$

in units of $\sec ^{-1}$, which should be compared with the scattering rate $\tau_{\mathrm{Ge}}^{-1}=3.67 \times 10^{-41} v^{4} \mathrm{sec}^{-1}$ obtained for $\mathrm{Ge}$ in the same limit. ${ }^{22}$

The calculation of the scattering rate for finite $\omega$, especially, for near-zone-boundary frequencies, requires knowledge of the frequency spectrum as well as of the polarization vectors over the entire Brillouin zone of crystals. It can be obtained on the basis of some specific lattice models and will be described in the next section.

\section{PHONON PROPERTIES IN GaAs AND InSb}

So far, a number of phenomenological approaches to the lattice vibrations of III-V compounds have been proposed. $^{23}$ Physically, the most appealing model for these compounds may be the BCM (Ref. 9), which contained, originally, six disposal parameters. Some results on the lattice vibrations in GaAs have been given by Lax and coworkers $^{5,24}$ based on the eight-parameter version of the BCM.

More traditional, well-known models exploited for the description of lattice vibrations may be the DDM (Refs. 25 and 26) and shell model ${ }^{27,28}(\mathrm{SM})$, although they require, to some extent, more adjustable parameters to give an excellent fit to the experimental dispersion curves. However, in order to obtain a quantitative fit to phonon frequencies, as well as elastic constants, piezoelectric constants, and dielectric constants, etc., we need to increase the number of parameters, e.g., short-range force constants, even in the framework of the BCM. In this respect we believe that the DDM and SM are as appealing as the BCM.

A crucial point we have found is that some lattice models show quite dissimilar behaviors to others regarding to the wavelength dependence of the vibrational amplitudes of constituent atoms. Typical examples may be the DDM with the deformable-bond approximation ${ }^{13,14}$ and the overlap-shell model with the valence-force-field concept, i.e., the VOSM. ${ }^{15-17}$ These models have been extensively applied to fit the experimental dispersion curves of crystals of zinc-blende structure. In the following we shall study the phonon properties of GaAs and InSb derived from these two models.

The dispersion curves of GaAs and InSb fitted by the DDM and VOSM are plotted in Figs. 1(a) and 1(b) together with the experimental data. ${ }^{11,12}$ For the DDM we have repeated the fitting procedure in order to obtain a better agreement of calculations with experiments, especially for the lower TA branch of GaAs. In this process, 14 param-
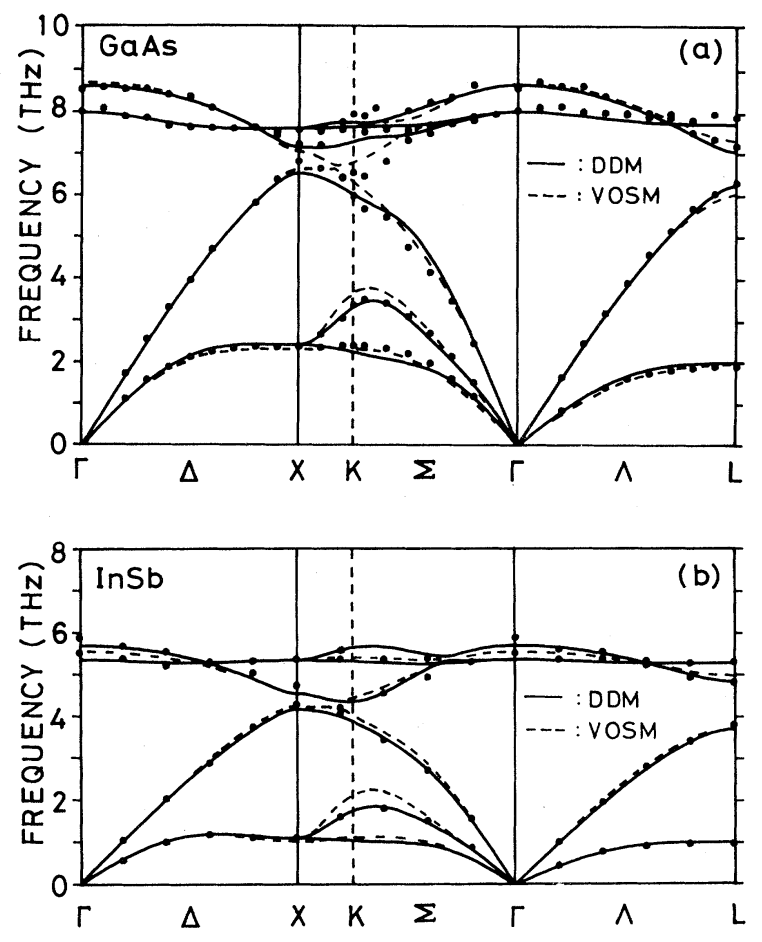

FIG. 1. Dispersion curves of (a) GaAs and (b) InSb along three principal directions calculated using the DDM (solid lines) and the VOSM (dashed lines). Experimental data are from Refs. 11 and 12 .

eters have been determined (we have assumed the local electric polarizability of the cation to be zero). For the VOSM, however, published values for ten parameters are employed. $^{29}$ The agreement of the calculations with experimental results is fairly good though some minor deviations are present. For example, the coincidence in the lower TA and LO branches in the [ $\xi \xi 0]$ direction of GaAs are found to still be insufficient for the DDM, whereas some deviation exists for the VOSM in the upper TA branch along the same direction. The latter discrepancy is also seen in InSb. Here we note that the situation for the lower TA branch of GaAs in the [ $\zeta \zeta 0]$ direction observed in the fit made using the DDM is quite similar to that made using the 14-parameter SM. ${ }^{12}$ Except for unaviodable disagreements which may stem from anharmonic effects, most of these are improved by increasing the number of parameters describing, for instance, the short-range forces. ${ }^{30}$

One-phonon densities of states calculated from these models are illustrated in Figs. 2(a) and 2(b). Thick lines show the ordinary one-phonon densities of states and thin lines represent the densities of states weighted by the squared amplitudes of the vibrations of $\mathrm{Ga}$ and $\mathrm{Sb}$ atoms which govern the scattering rates of phonons in GaAs and $\mathrm{InSb}$, respectively. As far as the dispersion relations and one-phonon densities of states are concerned, both the DDM and VOSM yield rather similar results and may be discriminated from each other only in the details of their structures. However, these two models are distinguished critically by the behaviors of the weighted density of 

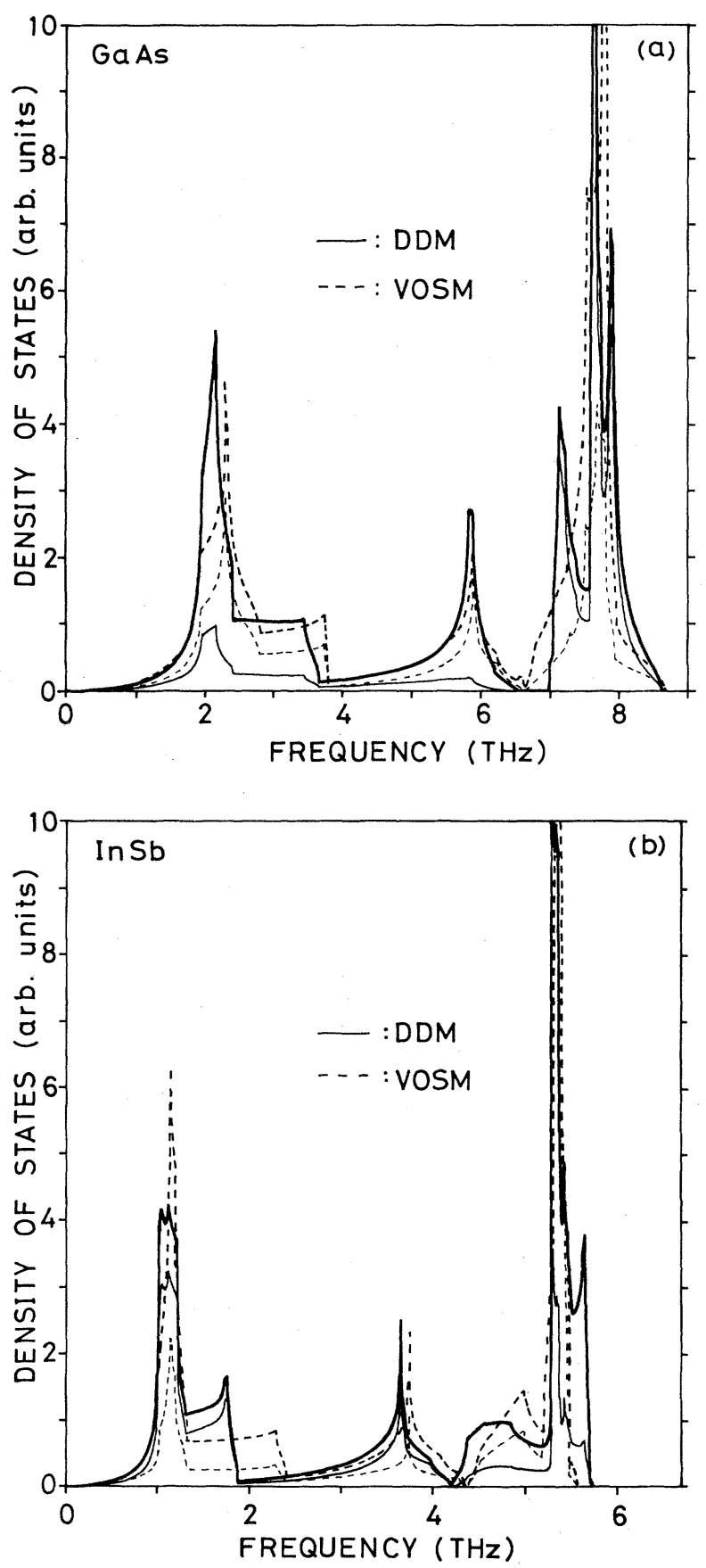

FIG. 2. Phonon densities of states of (a) GaAs and (b) InSb calculated using the DDM (solid lines) and the VOSM (dashed lines). Thick lines display ordinary one-phonon densities of states and thin lines represent the densities of states weighted by the squared amplitudes of (a) Ga motions and (b) Sb motions.

states. Compared to the ordinary one-phonon density of states, we recognize that in GaAs the weighted density of states derived from the DDM is reduced significantly for acoustic branches, whereas the corresponding reduction for optical branches is conspicuous in the result obtained by the VOSM. Just the opposite situation can be seen for InSb. From these results we expect, at near-zone-
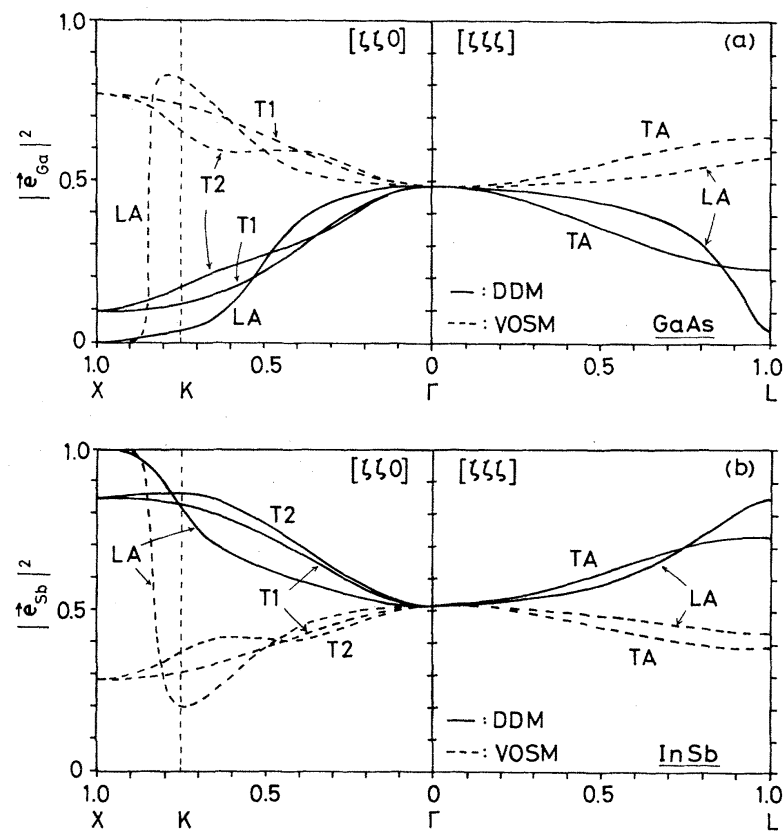

FIG. 3. Squared amplitudes of vibration of (a) Ga atoms and (b) $\mathrm{Sb}$ atoms versus reduced wave vector in the [ $\xi \xi 0]$ and $[\xi \xi \xi]$ directions. Solid and dashed lines show the results using the DDM and VOSM, respectively. T1 (T2) denotes the lower (upper) TA branch.

boundary frequencies of acoustic phonons, that the isotope scattering in GaAs (InSb) predicted by the DDM (VOSM) becomes considerably weaker than that predicted by the VOSM (DDM).

The origin of these behaviors of the weighted densities of states that we have found can be understood by studying the wavelength dependence of the vibrational amplitudes of constituent atoms. The squared amplitudes of $\mathrm{Ga}$ and $\mathrm{Sb}$ motions versus wave vector in the principal [ $\zeta \zeta \zeta]$ and [ $5 \zeta 0]$ directions of $\mathrm{GaAs}$ and $\mathrm{InSb}$ are plotted in Figs. 3(a) and 3(b), respectively. As the wave vector approaches the zone boundaries of these directions, the modulus of the atomic amplitude of the lighter element associated with the TA-mode vibrations decreases in the DDM but increases in the VOSM. These marked contrasts are also present in the TA branch of the [ $\xi 00]$ direction, although the DDM and VOSM provide very similar behaviors in regard to the vibrational amplitudes of the LA mode in this direction. Note that the scattering rates in GaAs and InSb are proportional, respectively, to the squared amplitudes of $\mathrm{Ga}$, and mainly to those of $\mathrm{Sb}$, atoms associated with the vibrations of initial phonons. Accordingly, Fig. 3 indicates to us that those factors describing the vibrations of initial phonons should act to enhance the scattering in GaAs (InSb) if the VOSM (DDM) is employed, but to suppress it if the DDM (VOSM) is assumed. This is indeed the case, as will be seen in the next section.

\section{NUMERICAL RESULTS ON RELAXATION TIME}

The phonon relaxation times versus frequency in GaAs in $\mathrm{InSb}$ up to the lowest zone-boundary frequencies of 
acoustic branches, i.e., the TA-phonon frequencies at point $L$ are shown in Figs. 4(a) and 4(b), respectively. As we see from Eq. (6) the isotope scattering in diatomic crystals depends on the propagation direction and the phonon mode. Figures 4(a) and 4(b) demonstrate the results for the TA phonons with the wave vector parallel to
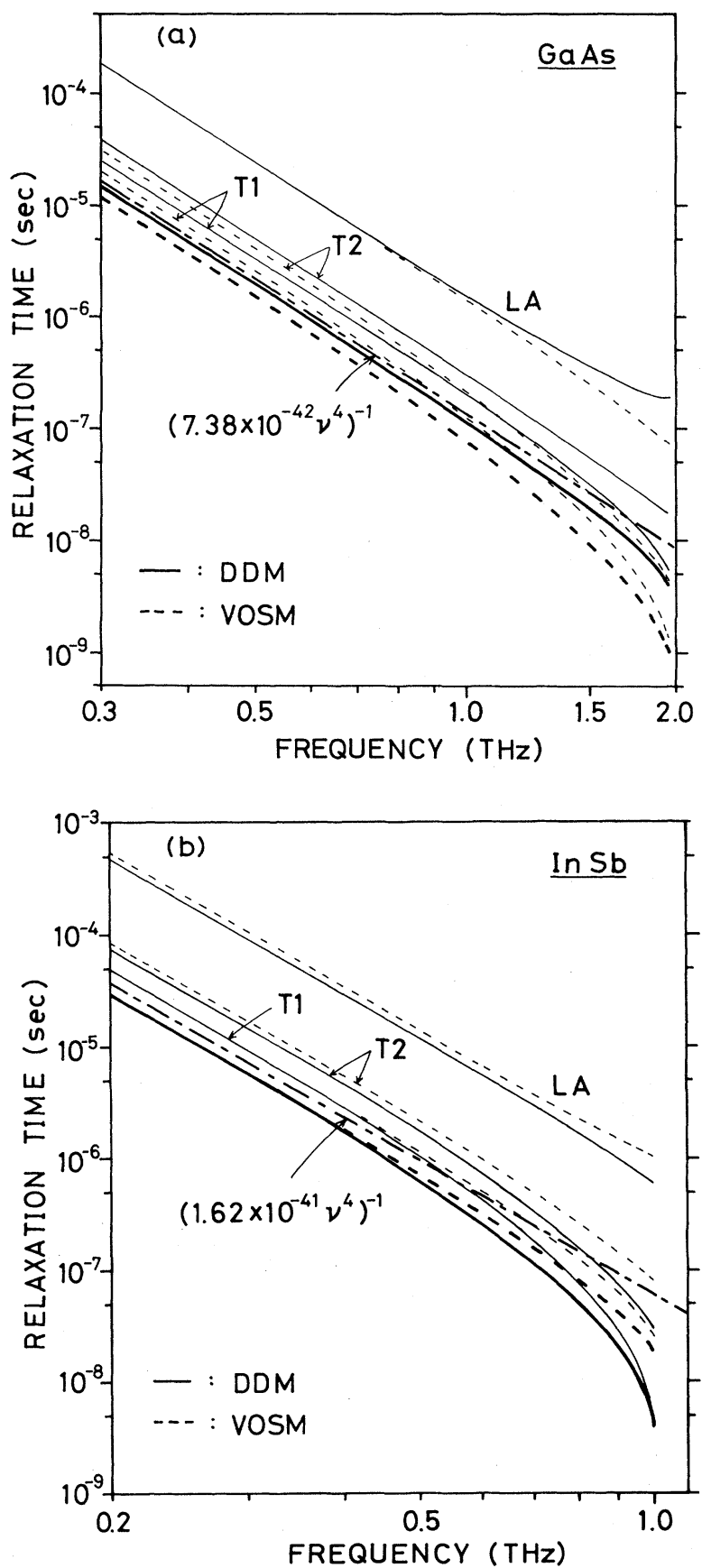

FIG. 4. Frequency dependence of relaxation time of TA phonons $(\overrightarrow{\mathrm{q}} \|[\zeta \zeta \zeta])$ up to the lowest zone-boundary frequency. (a) GaAs and (b) InSb. Solid and dashed lines represent the results using the DDM and VOSM, respectively. Thin lines show the contributions to the relaxation time of scattering into each acoustic branch. Long-dashed-short-dashed line shows the relaxation time extrapolated from low-frequency limit which shows the $\omega^{-4}$ behavior. the $[\xi \xi \zeta]$ direction. In order to comprehend explicitly the dispersive effects on the scattering the relaxation times proportional to $\omega^{-4}$ exptrapolated from the low-frequency limit [see Eq. (12)] are also plotted using longdashed-short-dashed lines.

Slight deviations from $\omega^{-4}$ lines of the calculated relaxation times in the low-frequency regions are due to inaccuracy of the values of elastic constants predicted by the employed models. As expected from Figs. 2 and 3 the departures of the relaxation times from $\omega^{-4}$ dependence near the zone-boundary frequencies are quite remarkable in those derived from the VOSM for GaAs and from the DDM for InSb. The ratio of the relaxation times deduced from these two models amounts to a factor of about 5 in both $\mathrm{GaAs}$ and InSb at zone-boundary frequencies.

Incidentally, it should be noted that the effects of the atomic amplitudes of initial phonons on the frequency dependence of the relaxation times [cf. Eq. (6)] are also observed from Fig. 4. They are measured by the amounts of deviations from the linearity of the lines representing the contribution to the relaxation times of the scattering into LA phonons. This is possible because the weighted density of terminal states of LA branch times $\omega^{2}$ is found to reveal almost exactly the $\omega^{4}$ behavior in the frequency ranges shown in Fig. 4, owing to the negligibly small dispersion of LA phonons at frequencies displayed.

Although the DDM and VOSM predict the relaxation times significantly different at near-zone-edge frequencies, they give rather similar magnitudes at frequencies, for instance, lower than $1 \mathrm{THz}$ for GaAs. According to these models the relaxation times of $1 \mu \mathrm{sec}$ are obtained at frequencies as low as 0.55 to $0.6 \mathrm{THz}$ for GaAs, which correspond to the wave number $|\vec{q}|=(0.25-0.29) \times q_{L}$, where $q_{L}$ is the wave number at point $L$ of the Brillouinzone boundary. At $1 \mathrm{THz}$ the relaxation time is about $10^{-7} \mathrm{sec}$ or shorter for GaAs. These results suggest that long-lived short-wavelength TA phonons reported by Ulbrich et al. $^{3}$ cannot be explained simply in terms of phonon lifetimes against isotope scattering.

In order to complete the analysis on the isotope effects we have studied further the anisotropy of the relaxation time which arises through $|\overrightarrow{\mathrm{e}}(\sigma \mid \overrightarrow{\mathrm{q}}, j)|^{2}$ of Eq. (6). This factor for the $\mathrm{Ga}$ atom is plotted in the (110) plane to estimate the anisotropy in the lower TA branch of GaAs. In the low-frequency limit no anisotropy exists due to Eq. (8). As the frequency increases the anisotropy increases. However, we find it rather small, e.g., at $1.5 \mathrm{THz}$ the variation of squared amplitude of $\mathrm{Ga}$ motion in GaAs is about $10 \%$ in the DDM and it is much smaller in the VOSM. Then, the anisotropy of the relaxation time is practically irrelevant for GaAs as far as the frequencies as low as $1.5 \mathrm{THz}$ are concerned.

\section{CONCLUDING REMARKS}

We have studied the isotope scattering of near-zoneboundary acoustic phonons in GaAs and InSb by assuming that these compounds are composed of normal elements with naturally occurring distributions of isotopes. The scattering rate of phonons depends in an important way on the vibrational amplitudes of isotopically impure 
elements. The wavelength dependence of these amplitudes relies sensitively on lattice models and even on the values of parameters of the specific model. As a result we expect that some models predict the scattering rates with strong departure from $\omega^{4}$ behavior near zone boundaries, exhibiting large dispersive effects, though others predict a weak departure. This remarkable feature reflects the essential difference in the nature of interatomic forces characteristic of each model. Therefore, the experimental study of the isotope scattering of large-wave-vector phonons is also of great interest in that it may provide a test of the validity of these lattice models.

Through this work we have illustrated the results based on the DDM and VOSM. At phonon frequencies higher than $1 \mathrm{THz}$ these models yield, for GaAs, relaxation times much shorter than $1 \mu \mathrm{sec}$. This implies that it is hard to explain the experimentally observed long-lived dispersive phonons naively in terms of the isotope scattering incorporating the dispersion unless the experiment by Ulbrich et $a{ }^{3} .^{3}$ has been conducted with an isotopically enriched sample of GaAs. At present there seems to be no mechanism which may account for the result of Ulbrich et $a l^{3}$ as far as appreciable isotope scattering occurs in sample. ${ }^{31}$

More recently, using the ballistic-phonon-imaging technique, Wolfe and Northrop ${ }^{32}$ have carried out a systematic study of spatial and temporal behaviors of a photoproduced heat pulse of GaAs. They have found that the dispersive shift in the phonon-focusing pattern which evidences the ballistic propagations of near-zone-boundary phonons is observed only in a thin sample (about $250 \mu \mathrm{m}$ thick), suggesting the short mean free path of highly dispersive phonons with a frequency of about $1 \mathrm{THz}$. The relaxation times we have obtained for GaAs lead to a mean free path of about $300 \mu \mathrm{m}$ for $1-\mathrm{THz}$ TA phonons. This result may be in accord with the experiment by Wolfe and Northrop. ${ }^{32}$

\section{ACKNOWLEDGMENTS}

The author would like to thank Professor T. Sakuma for a critical reading of the manuscript. This work is partially supported by a Grant-in-Aid for Scientific Research from the Ministry of Education, Science and Culture, Japan.
${ }^{1}$ Proceedings of the International Conference on Phonon Physics, edited by W. Bron [J. Phys. (Paris) Colloq. 42, C6 (1981)].

${ }^{2}$ Phonon Scattering in Condensed Matter, edited by W. Eisenmenger (Springer, Berlin, 1984).

${ }^{3}$ R. G. Ulbrich, V. Narayanamurti, and M. A. Chin, Phys. Rev. Lett. 45, 1432 (1980).

${ }^{4}$ R. Orbach and L. A. Vredevoe, Physics (N.Y.) 1, 91 (1964).

${ }^{5}$ H. J. Maris, Phys. Lett. 17A, 228 (1965).

${ }^{6}$ M. Lax, P. Hu, and V. Narayanamurti, Phys. Rev. B 23, 3095 (1981).

${ }^{7}$ P. G. Klemens, Proc. Phys. Soc. London, Sect. A 68, 1113 (1955).

${ }^{8}$ M. Lax, V. Narayanamurti, P. Hu, and W. Weber, J. Phys. (Paris) Colloq. 42, C6-161 (1981).

${ }^{9}$ K. C. Rustagi and W. Weber, Solid State Commun. 18, 673 (1976).

${ }^{10}$ R. S. Leigh, B. Szigeti, and V. K. Tewary, Proc. R. Soc. London 320, 505 (1971).

${ }^{11}$ D. L. Price, J. M. Rowe, and R. M. Nicklow, Phys. Rev. B 3, 1268 (1971).

${ }^{12}$ G. Dolling and J. L. Waugh, in Lattice Dynamics, edited by R. F. Wallis (Pergamon, London, 1965), p. 19.

${ }^{13}$ K. Kunc, M. Balkanski, and M. Nusimovici, Phys. Rev. B 12, 4363 (1975).

${ }^{14}$ K. Kunc, M. Balkanski, and M. Nusimovici, Phys. Status Solidi B 71, 341 (1975); 72, 229 (1975).

${ }^{15} \mathrm{~K}$. Kunc and $\mathrm{H}$. Bilz, in Proceedings of the International Conference on Neutron Scattering, edited by R. M. Moon (ERDA Conf-760601-P2, Oak Ridge, Tennessee, 1976), p. 195.

${ }^{16}$ K. Kunc and H. Bilz, Solid State Commun. 19, 1027 (1976).

${ }^{17}$ P. H. Borcherds and K. Kunc, J. Phys. C 11, 4145 (1978).

${ }^{18}$ S. Tamura, Phys. Rev. B 27, 858 (1983).

${ }^{19}$ A. A. Maradudin, E. W. Montroll, G. H. Weiss, and I. P. Ipa- tova, Theory of Lattice Dynamics in the Harmonic Approximation, 2nd ed. (Academic, New York, 1971).

${ }^{20}$ T. B. Bateman, H. J. McSkimin, and J. M. Whelan, J. Appl. Phys. 30, 544 (1959).

${ }^{21}$ L. J. Slutsky and C. W. Garland, Phys. Rev. 113, 167 (1959).

${ }^{22}$ For InSb, $\widetilde{g}=2.04 \times 10^{-5}$, which is smaller than $\widetilde{g}=4.58$ $\times 10^{-5}$ for GaAs, but in the low-frequency limit the density of states in InSb is much larger than that in GaAs, leading to a value of $\tau_{\text {InSb }}^{-1}$ larger than $\tau_{\text {GaAs. }}^{-1}$.

${ }^{23} \mathrm{H}$. Bilz and W. Kress, Phonon Dispersion Relations in Insulators (Springer, Berlin, 1979).

${ }^{24}$ M. Lax, V. Narayanamurti, R. C. Fulton, R. Bray, K. T. Tsen, and K. Wan, in Phonon Scattering in Condensed Matter, Ref. 2, p. 133.

25J. R. Hardy, Philos. Mag. 4, 1278 (1959).

${ }^{26}$ A. M. Karo and J. R. Hardy, Phys. Rev. 129, 2024 (1963).

${ }^{27}$ B. G. Dick and A. W. Overhauser, Phys. Rev. 112, 90 (1958).

${ }^{28}$ W. Cochran, Proc. R. Soc. London, Ser. A 253, 260 (1959).

${ }^{29}$ For the VOSM we have also tried readjusting the parameters in order to obtain a better fit to elastic-constants etc. In this course, we have found that this model predicts small values of 3-4 for dielectric constants even when they are included in the fitting process.

${ }^{30} \mathrm{We}$ have confirmed this by introducing parameters describing the short-range forces up to fifth-nearest neighbors in the framework of the DDM. Excellent agreements of dispersion curves (e.g., for GaAs $1 / n \sum_{i=1}^{n}\left|v_{i}^{\text {fit }}-v_{i}^{\text {expt }}\right| / v_{i}^{\text {expt }}<2.0 \%$, $n=72$ being the total number of fitted frequencies) are obtained, with a small deviation in the LO branch for which anharmonic effects should be large.

${ }^{31}$ M. Lax, V. Narayanamurti, R. G. Ulbrich, and N. Holzwarth, in Phonon Scattering in Condensed Matter, Ref. 2, p. 103.

32J. P. Wolfe and G. A. Northrop, in Phonon Scattering in Condensed Matter, Ref. 2, p. 100. 